\title{
Deficiency of eNOS exacerbates early-stage NAFLD pathogenesis by changing the fat distribution
}

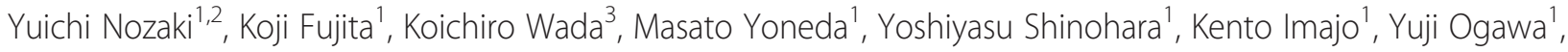 \\ Takaomi Kessoku', Makoto Nakamuta ${ }^{4}$, Satoru Saito ${ }^{1}$, Naohiko Masaki $^{5}$, Yoji Nagashima ${ }^{6,7}$, Yasuo Terauchi $^{8}$ \\ and Atsushi Nakajima ${ }^{1 *}$
}

\begin{abstract}
Background: Although many factors and molecules that are closely associated with non-alcoholic fatty liver disease (NAFLD)/non-alcoholic steatohepatitis (NASH) have been reported, the role of endothelial nitric oxide synthase (eNOS)-derived nitric oxide (NO) in the pathogenesis of NAFLD/NASH remains unclear. We therefore investigated the role of eNOS-derived NO in NAFLD pathogenesis using systemic eNOS-knockout mice fed a high-fat diet.

Methods: eNOS-knockout and wild-type mice were fed a basal diet or a high-fat diet for 12 weeks. Lipid accumulation and inflammation were evaluated in the liver, and various factors that are closely associated with NAFLD/NASH and hepatic tissue blood flow were analyzed.

Results: Lipid accumulation and inflammation were more extensive in the liver and lipid accumulation was less extensive in the visceral fat tissue in eNOS-knockout mice, compared with wild-type mice, after 12 weeks of being fed a high-fat diet. While systemic insulin resistance was comparable between the eNOS-knockout and wild-type mice fed a high-fat diet, hepatic tissue blood flow was significantly suppressed in the eNOS-knockout mice, compared with the wild-type mice, in mice fed a high-fat diet. The microsomal triglyceride transfer protein activity was down-regulated in eNOS-knockout mice, compared with wild-type mice, in mice fed a high-fat diet.
\end{abstract}

Conclusions: A deficiency of eNOS-derived NO may exacerbate the early-stage of NASH pathogenesis by changing the fat distribution in a mouse model via the regulation of hepatic tissue blood flow.

Keywords: NO, NAFLD, Hepatic tissue blood flow, Fat distribution, Obese mice

\section{Background}

Non-alcoholic fatty liver disease (NAFLD) is one of the most important causes of chronic liver disease and is associated with systemic insulin resistance (IR) and metabolic syndrome. NAFLD includes non-alcoholic fatty liver (NAFL) and non-alcoholic steatohepatitis (NASH); NAFL represents the first phase of NASH, which is characterized by steatosis, and can then develop into fatty liver disease with associated inflammation [1].

\footnotetext{
* Correspondence: nakajima-tky@umin.ac.jp

'Department of Gastroenterology, Yokohama City University Graduate School of Medicine, 3-9 Fuku-ura, Kanazawa-ku, 236-0004 Yokohama, Kanagawa, Japan

Full list of author information is available at the end of the article
}

$\mathrm{NASH}$ is thought to lead to liver fibrosis, cirrhosis, and hepatocellular carcinoma, resulting in increased morbidity and mortality. The pathogenesis of NAFLD remains to be clarified fully.

We previously reported that several potential mechanisms are involved in the pathogenesis of NAFLD [2-4]. However, other factors associated with NAFLD progression remain to be determined.

Nitric oxide $(\mathrm{NO})$ is a gas produced by nitric oxide synthase (NOS) enzyme [5], and three major isoforms of NOS are known to exist: neuronal NOS (nNOS), inducible NOS (iNOS), and endothelial NOS (eNOS). eNOSderived $\mathrm{NO}$ is known to have important endothelial functions, including the regulation of vascular tone and 
regional blood flow [6]. On the other hand, liver steatosis has been shown to be associated with sinusoidal narrowing and a reduction in sinusoidal flow; furthermore, microvascular changes may contribute to progressive liver injury in metabolic forms of steatohepatitis [7]. Chronic NOS inhibition has been reported to accelerate NAFLD progression [8]; however, the roles of eNOS and generated $\mathrm{NO}$ in the pathogenesis of NAFLD/NASH have not yet been fully elucidated in eNOS knockout (eNOS-/-) mice fed a high-fat diet (HFD).

In this study, we investigated the role of eNOS-derived $\mathrm{NO}$ in the pathogenesis of NAFLD using systemic eNOS-knockout mice fed an HFD.

\section{Methods}

\section{Animal treatment and procedures}

We purchased a C57BL/6J backcrossed C57BL/6JNos3tm1Lau colony from Jackson Labs (Bar Harbor, ME 04609, USA) to create male congenic wild-type (eNOS $+/+)$ and eNOS knockout (eNOS-/-) mice. Mice were allowed free access to food and tap water throughout the acclimatization and experimental periods.

Beginning at 6 weeks of age, each strain of mice was fed a basal diet (BD) or an HFD. Thirty-two mice were divided into 4 groups: (1) eNOS+/+ and $\mathrm{BD}$; (2) eNOS-/and BD; (3) eNOS+/+ and HFD; and (4) eNOS-/- and HFD. After a 12-week experimental period, eight mice in each group were examined after being subjected to an overnight fast. The HFD consisted of high-fat diet 32, containing $20 \%$ protein, $60 \%$ fat, and $20 \%$ carbohydrate, in powdered form from Japan CLEA (Tokyo, Japan), while the BD consisted of MF, containing $22 \%$ protein, $6 \%$ fat, and $47 \%$ carbohydrate, from Oriental Yeast Co., Ltd. (Tokyo, Japan). The precise contents of these feeds have been described previously [4]. All the animals were treated humanely according to the guidelines of the National Institutes of Health and the AERIBBRI Animal Care and Use Committee. The animal protocols were approved by the Yokohama City University Medical School guidelines for the care and use of laboratory animals. This study was conducted by the approval of the institutional review board at the Yokohama City University Medical School.

\section{Measurement of plasma and serum biochemical markers}

Serum alanine aminotransferase (ALT) was measured using Spotchem SP-4410 (Arklay Co., Kyoto, Japan). The total serum cholesterol (Chol) and triglyceride (TG) levels were analyzed using an online dual-enzymatic method for the simultaneous quantification of cholesterol and TG using high-performance liquid chromatography according to a previously described procedure $[4,9,10]$. The fasting plasma glucose level and the serum levels of fasting insulin, leptin, adiponectin, and non-esterified fatty acid (NEFA) were determined using a Glutest Pro kit (Sanwa Kagaku Kenkyusyo Co., Nagoya, Japan), an Ultra Sensitive Insulin ELISA kit (Biochemical Research Laboratory, Morinaga Milk Industry Co., Tokyo, Japan), an ELISA Mouse Leptin kit (Biochemical Research Laboratory, Morinaga Milk Industry Co., Tokyo, Japan), a Mouse/Rat Adiponectin ELISA kit (Otsuka Pharmaceutical Co., Tokyo, Japan), and a NEFA C-Test kit (Wako Pure Chemical Industries Co., Osaka, Japan), respectively. The blood IR was estimated using the homeostasis model assessment of IR (HOMA-IR) and the following equation: $\mathrm{IR}$ = fasting plasma glucose level $(\mathrm{mg} /$ $\mathrm{dL}) \times$ fasting serum insulin level $(\mathrm{ng} / \mathrm{mL}) / 22.5$.

\section{Insulin tolerance test (ITT)}

Mice were fed ad libitum prior to being subjected to a fast immediately before the start of the study. The mice were intraperitoneally challenged with $0.75 \mathrm{mU} / \mathrm{g}$ body weight of human insulin (Novolin R; Novo Nordisk, Denmark). Blood samples were then collected to measure the glucose levels at $0,30,60,80,100$, and $120 \mathrm{~min}$ after the insulin injection [11].

\section{Measurement of liver triglyceride content}

The liver samples were homogenized in $50 \mathrm{mM}$ Tris/ $\mathrm{HCl}$ buffer, pH7.4, containing $150 \mathrm{mM} \mathrm{NaCl}, 1 \mathrm{mM}$ EDTA, and $1 \mathrm{mM}$ PMSF. The liver TG levels were analyzed enzymatically using a diagnostic kit (Infinity ${ }^{\mathrm{Tm}}$; Thermo DMA, Arlington, TX, USA).

\section{Liver MTP activity assay}

MTP activity was measured using an MTP assay kit (Roar Biochemical, New York, NY, USA), according to a previously described method [12, 13].

\section{CT scanning}

In the 12-week model, the abdomen of each mouse was scanned prior to sacrifice using an X-ray computed tomography (CT) system (eXplore Locus; GE Healthcare Bio-Sciences GK, Tokyo, Japan) developed for small experimental animals [14]. The mice were anesthetized for the duration of the scan, and non-contrast CT scanning was performed.

\section{Measurement of liver/spleen ratio of CT values and visceral fat volume}

The hepatic attenuation index was obtained using noncontrast CT images to determine the liver-to-spleen attenuation ratio [15]. The inverse of the liver-to-spleen density ratio was regarded as an indicator of the degree of fat infiltration in the liver [16]. The visceral fat volume was quantified using CT imaging $[17,18]$. 


\section{Determination of Hepatic Tissue Blood Flow}

At 12 weeks, each mouse was anesthetized and a laparotomy was conducted to determine the hepatic tissue blood flow at several points located $1 \mathrm{~mm}$ from the edge of the right or left lobe using a noncontact-type laser Doppler blood flow meter (OMEGAFLO FLO-N1; OMEGAWAVE Inc., Tokyo, Japan) [19, 20]. The measurements were performed at 3 different points in each of the right and left lobes. The mean of the three values for each lobe was calculated, and the resulting values were expressed as a percentage of the value obtained in the $e N O S+/+$ and BD group.

\section{Liver histopathological and immunohistochemical evaluations}

Liver samples were excised and embedded in Tissue-Tek OCT compound (Sakura Finetek USA Inc., Torrance, CA, USA) and paraffin for the histological analysis. Formalinfixed and paraffin-embedded sections were processed routinely using hematoxylin and eosin $(\mathrm{H} \& \mathrm{E})$, myeloperoxidase (MPO), naphthol AS-D chloroacetate esterase [21] and Sirius-red. To evaluate the fat deposition, the OCT-embedded samples were stained with oil-red O.

\section{Liver histology and scoring systems}

All the histopathological findings were scored by the same pathologists (Y.N. and S.M.), who were unaware of the treatments that the animals had received. The histological features were grouped into 3 broad categories (steatosis, inflammation, and fibrosis) to enable the use of the NAFLD activity score (NAS) [22]. The evaluation protocol, which has been described previously [23], is detailed in Table 3.

\section{RNA isolation and reverse transplantation}

Total RNA was isolated from the samples using an RNeasy Mini kit (Qiagen GmbH, Hilden, Germany; Cat No. 74126); reverse transcription was then performed to produce cDNA using a TaqMan Gold RT-PCR Kit (Applied Biosystems, Foster City, CA, USA) according to the manufacturer's instructions, as previously described [4].

\section{Quantification of gene expressions using real-time RT-PCR} The hepatic mRNA levels of several markers, as well as the housekeeping gene $\beta$-actin, in the liver tissues were determined using fluorescence-based real-time RT-PCR and an ABI PRISM 7700 Sequence Detection System (Applied Biosystems, Foster City, CA, USA). Real-time RT-PCR was performed using the TaqMan and Power SYBR Green PCR Master Mix reagent, according to the manufacturer's instructions (Applied Biosystems, Foster City, CA, USA). The values were normalized to the expression level of the endogenous control, $\beta$-actin. The gene expression ratio was determined using data from the eNOS+/+ and BD group mice as the control group. The probe and primer pair specific for $\beta$-actin were purchased from Applied Biosystems. The primer sequences of sterol regulatory element binding protein-1c (SREBP-1c), peroxisome proliferators-activated receptor alpha1 (PPAR- $\alpha 1), \mathrm{nNOS}$, and iNOS are listed in Table 1.

\section{Statistical analysis}

The statistical analyses were performed using SPSS for Windows, version 12 (IBM Co. Armonk, NY, USA). All the results were expressed as the mean \pm SEM. Statistical comparisons were made using the Student $t$-test or Scheffe's method after an analysis of variance (ANOVA). A value of $P<0.05$ was considered statistically significant.

\section{Results}

HFD feeding induced the phenotype of metabolic syndrome and the pathogenesis of early-stage NASH

After 12 weeks of HFD feeding, the eNOS+/+ and HFD mice had significantly higher body weights, liver weights, and visceral fat weights than the control mice $(e N O S+/+$ and $\mathrm{BD}$ ) (Table 2). The $e N O S+/+$ and HFD mice showed signs of insulin resistance and dyslipidemia (Table 2 and Fig. 4a), thus demonstrating the phenotype of metabolic syndrome. Furthermore, hepatic steatosis and very mild inflammation were observed in the $e N O S+/+$ and HFD mice, which were considered to have early-stage $\mathrm{NASH}$ (Table 3 and Figs. 1a, 2a-d). The reason for the negative findings of the liver tissue MPO staining examination might be related to the model used in this study for earlystage NASH, which showed very mild inflammation, such as very small inflammatory foci detected in the $H-E$ stained samples (Fig. 2d). No apparent signs of liver fibrosis were observed in any of the groups (Fig. 2e).

\section{Deficiency of eNOS-derived NO increased lipid deposits in the liver and decreased the visceral fat volume in HFD-fed mice}

In the NAFLD/NASH model, an increase in the body weight was observed after 12 weeks of HFD feeding, and no significant difference was observed between the eNOS $+/+$ and HFD mice and the eNOS-/- and HFD mice (Table 2). The eNOS-/- and HFD mice had a significantly higher liver weight and a significantly lower visceral fat weight than then $e N O S+/+$ and HFD mice; furthermore, the $e N O S$ - / - and HFD mice had a significantly higher liver weight/body weight ratio and a significantly lower visceral fat weight/body weight ratio, compared with the $e \mathrm{NOS}+/+$ and HFD mice (Table 2).

As an indicator of the degree of fat infiltration in the liver, the liver-to-spleen attenuation ratio obtained using non-contrast CT images showed that hepatic steatosis was significantly severer in the eNOS-/- and HFD mice than in the $e N O S+/+$ and HFD mice (Fig. 1a). 
Table 1 Primer sequences used for real-time PCR analysis

\begin{tabular}{lll}
\hline Gene & Primer sense & Primer antisense \\
\hline SREBP-1C & CAGCTATTGGCCTTCCTCAG & CCTGGACCATTTTAGCCTCA \\
PPAR-a & GTCCTCAGTGCTTCCAGAGG & GGTCACCTACGAGTGGCATT \\
nNOS & TCCTAAATCCAGCCGATCGA & TCATGGTTGCCAGGGAAGAC \\
iNOS & AGAGAGATCCGATTAGAGTCTTGGT & TGACCCGTGAAGCCATGAC \\
\hline
\end{tabular}

The visceral fat volume as quantified using CT imaging was significantly lower in the eNOS-/- and HFD mice than in the eNOS+/+ and HFD mice (Fig. 1b).

An analysis of the parameters associated with metabolic syndrome showed no significant differences between the $e N O S+/+$ and HFD mice and the $e N O S-/-$ and HFD mice when the fasting plasma glucose level, fasting insulin level, HOMA-IR, serum TG level, serum NEFA level, and serum leptin level were compared. However, the serum cholesterol level was significantly higher in the eNOS-/and HFD mice than in the eNOS+/+ and HFD mice (Table 2).

\section{Deficiency of eNOS-derived NO exacerbated the pathogenesis of early-stage NASH in HFD-fed mice}

Pathological examination of the liver showed that the eNOS-/- and HFD mice had severer hepatic steatosis and inflammation than the $e N O S+/+$ and HFD mice (Fig. 2a and 2c). The liver TG content and the serum ALT levels were significantly higher in the eNOS-/- and HFD mice than in the eNOS+/+ and HFD mice (Fig. 2b and Table 2). The liver tissue MPO and Sirius red staining examinations showed no significant positive findings
(Fig. 2d and 2e), and the additional liver tissue naphthol AS-D chloroacetate esterase staining examination showed negative findings (data not shown). An analysis of the histological scores for the livers using NAS showed that the scores for the steatosis grade were significantly higher in the $e N O S$ - / - and HFD mice than in the eNOS+/+ and HFD mice; these results were consistent with the results of the CT scan examination. Furthermore, the scores for lobular inflammation and liver cell injury were significantly higher for the eNOS-/- and HFD mice than for the eNOS+/t and HFD mice (Table 3).

\section{Deficiency of eNOS-derived NO decreased the hepatic tissue blood flow}

Measurement of the hepatic tissue blood flow using a noncontact-type laser Doppler blood flow meter showed that a deficiency of eNOS-derived NO significantly decreased the hepatic tissue blood flow in both hepatic lobes under each dietary condition (Fig. 3). HFD feeding was also shown to significantly decrease the hepatic blood flow, since the value for the eNOS+/+ and HFD

Table $\mathbf{2}$ Characteristics of mice in the 12-week model

\begin{tabular}{|c|c|c|c|c|}
\hline & \multicolumn{4}{|c|}{ 12-week model } \\
\hline & eNOS+/+BD & eNOS-/-BD & eNOS+/+HFD & eNOS-/-HFD \\
\hline Number of animals & 8 & 8 & 8 & 8 \\
\hline Body weight (g) & $28.1 \pm 1.9$ & $27.2 \pm 2.4$ & $42.8 \pm 3.1^{*}$ & $41.7 \pm 2.4$ \\
\hline Liver weight (g) & $1.2 \pm 0.1$ & $1.2 \pm 0.2$ & $2.3 \pm 0.6^{*}$ & $2.8 \pm 0.5 \S$ \\
\hline Visceral fat weight (g) & $0.6 \pm 0.1$ & $0.7 \pm 0.3$ & $2.0 \pm 0.3^{*}$ & $1.2 \pm 0.3 \S$ \\
\hline Liver weight/body weight ratio (\%) & $4.2 \pm 0.3$ & $4.3 \pm 0.6$ & $5.2 \pm 1.0^{*}$ & $6.7 \pm 1.0 \S$ \\
\hline Visceral fat weight/body weight ratio (\%) & $2.1 \pm 0.4$ & $2.4 \pm 1.0$ & $4.7 \pm 1.0^{*}$ & $3.0 \pm 0.6 \S$ \\
\hline Serum ALT (IU/L) & $9.2 \pm 0.8$ & $17.5 \pm 9.3$ & $74.8 \pm 41.4^{*}$ & $172.8 \pm 58.2 \S$ \\
\hline Fasting glucose (mg/dL) & $89.6 \pm 8.0$ & $85.7 \pm 20.6$ & $151.8 \pm 36.4^{*}$ & $135.8 \pm 32.3$ \\
\hline Fasting insulin (ng/mL) & $0.72 \pm 0.41$ & $0.57 \pm 0.72$ & $1.63 \pm 0.81^{*}$ & $2.10 \pm 0.74$ \\
\hline HOMA-IR & $2.9 \pm 1.8$ & $2.0 \pm 2.5$ & $11.0 \pm 6.7^{*}$ & $13.3 \pm 6.6$ \\
\hline Serum cholesterol (mg/dL) & $90.5 \pm 3.8$ & $85.1 \pm 4.9$ & $174.8 \pm 22.9^{*}$ & $263.0 \pm 28.5 \S$ \\
\hline Serum TG (mg/dL) & $24.8 \pm 6.8$ & $26.0 \pm 5.6$ & $31.3 \pm 5.9^{*}$ & $33.8 \pm 8.2$ \\
\hline Serum NEFA (mEq/L) & $1.2 \pm 0.0$ & $1.1 \pm 0.2$ & $1.8 \pm 0.1^{*}$ & $1.7 \pm 0.3$ \\
\hline Serum leptin (ng/mL) & $3.9 \pm 2.0$ & $3.5 \pm 2.7$ & $30.2 \pm 4.4^{*}$ & $31.6 \pm 22.9$ \\
\hline
\end{tabular}

$B D$ basal diet, HFD high-fat diet, HOMA-IR homeostasis model assessment of insulin resistance, TG triglyceride, NEFA nonesterified fatty acid Data are expressed as the mean \pm SEM

Significant differences exist between * ${ }^{*} \mathrm{NOS}+/+$ and BD vs. eNOS+/+ and HFD; §eNOS+/+ and HFD vs. eNOS-/- and HFD for listed parameters at the $P<.05$ level 
Table 3 Histological Scores of Livers using the NAFLD activity score (NAS) ${ }^{11)}$

\begin{tabular}{|c|c|c|c|c|c|c|}
\hline \multirow{3}{*}{ Item } & \multirow{3}{*}{ Definition } & \multirow{3}{*}{ Score } & \multicolumn{4}{|c|}{ 12-week model } \\
\hline & & & eNOS+/+ & eNOS-/- & eNOS+/+ & eNOS- $/-$ \\
\hline & & & $\mathrm{BD}$ & $\mathrm{BD}$ & HFD & HFD \\
\hline \multicolumn{7}{|l|}{ Steatosis } \\
\hline \multirow[t]{6}{*}{ Grade } & Parenchymal involvement & & & & & \\
\hline & $<5 \%$ & 0 & 8 & 8 & 0 & 0 \\
\hline & $5 \%-33 \%$ & 1 & 0 & 0 & 6 & 2 \\
\hline & $33 \%-66 \%$ & 2 & 0 & 0 & 2 & 6 \\
\hline & $>66 \%$ & 3 & 0 & 0 & 0 & 0 \\
\hline & & Average & 0.00 & 0.00 & $1.25^{*}$ & $1.75 \S$ \\
\hline \multicolumn{7}{|l|}{ Inflammation } \\
\hline \multirow[t]{5}{*}{ Lobular inflammation } & Assessment of all inflammatory foci & & & & & \\
\hline & No foci & 0 & 8 & 8 & 5 & 1 \\
\hline & $<2$ foci per $200 \times$ field & 1 & 0 & 0 & 3 & 7 \\
\hline & 2-4 foci per $200 \times$ field & 2 & 0 & 0 & 0 & 0 \\
\hline & $>4$ foci per $200 \times$ field & 3 & 0 & 0 & 0 & 0 \\
\hline \multirow[t]{5}{*}{ Liver cell injury } & Ballooning & & & & & \\
\hline & None & 0 & 8 & 8 & 0 & 0 \\
\hline & Few balloon cells & 1 & 0 & 0 & 8 & 8 \\
\hline & Many cells/prominent ballooning & 2 & 0 & 0 & 0 & 0 \\
\hline & & Average & 0.00 & 0.00 & $1.38^{*}$ & $1.88 \S$ \\
\hline \multicolumn{7}{|l|}{ Fibrosis } \\
\hline \multirow[t]{7}{*}{ Stage } & Method of Brunt & & & & & \\
\hline & None & 0 & 8 & 8 & 8 & 8 \\
\hline & Perivenular/perisinusoidal fibrosis & 1 & 0 & 0 & 0 & 0 \\
\hline & Combine pericellular portal fibrosis & 2 & 0 & 0 & 0 & 0 \\
\hline & Septal/bridging fibrosis & 3 & 0 & 0 & 0 & 0 \\
\hline & Cirrhosis & 4 & 0 & 0 & 0 & 0 \\
\hline & & Average & 0.00 & 0.00 & 0.00 & 0.00 \\
\hline
\end{tabular}

$B D$ basal diet, $H F D$ high fat diet

Significant differences exist between ${ }^{*}$ eNOS $+/+$ and BD vs. eNOS+/+ and HFD; §eNOS+/+ and HFD vs. eNOS-/- and HFD for listed parameters at the $P<.05$ level

mice was significantly lower than that of the eNOS+/+ and $\mathrm{BD}$ mice (Fig. 3).

\section{Comparison of the mechanism associated with the pathogenesis of NAFLD between wild-type and eNOS-knockout mice under HFD conditions}

The ITT results at 12 weeks showed no significant difference in the systemic response to insulin injection between the eNOS+/+ and HFD mice and the eNOS-/and HFD mice, while it was significantly lower in the eNOS+/+ and HFD mice than in the $e N O S+/+$ and BD mice (Fig. 4a).

An analysis of the liver mRNA expression levels showed that no significant differences in the liver mRNA levels of SREBP-1c and PPAR- $\alpha 1$ were observed between the $e N O S$ $+/+$ and HFD mice and the eNOS-/- and HFD mice. Furthermore, the liver MTP activity level was significantly down-regulated in the eNOS-/- and HFD mice, compared with the eNOS+/+ and HFD mice (Fig. 4b). As for the liver mRNA levels of other isoforms of NOS, no significant differences in the levels of either nNOS or iNOS were observed between the $e N O S+/+$ and HFD mice and the eNOS-/- and HFD mice (data not shown).

\section{Discussion}

NAFLD/NASH is presently the most common chronic liver disorder, displaying a wide spectrum of liver damage ranging from simple steatosis to steatohepatitis, advanced fibrosis, and cirrhosis. NAFLD/NASH is a complex disease with no simple causes. A new disease model has been proposed suggesting that multiple hits may act in parallel, resulting in liver inflammation; this model is known as the multiple parallel hits hypothesis [24]. A relationship between fatty liver changes and hepatic microcirculation has 

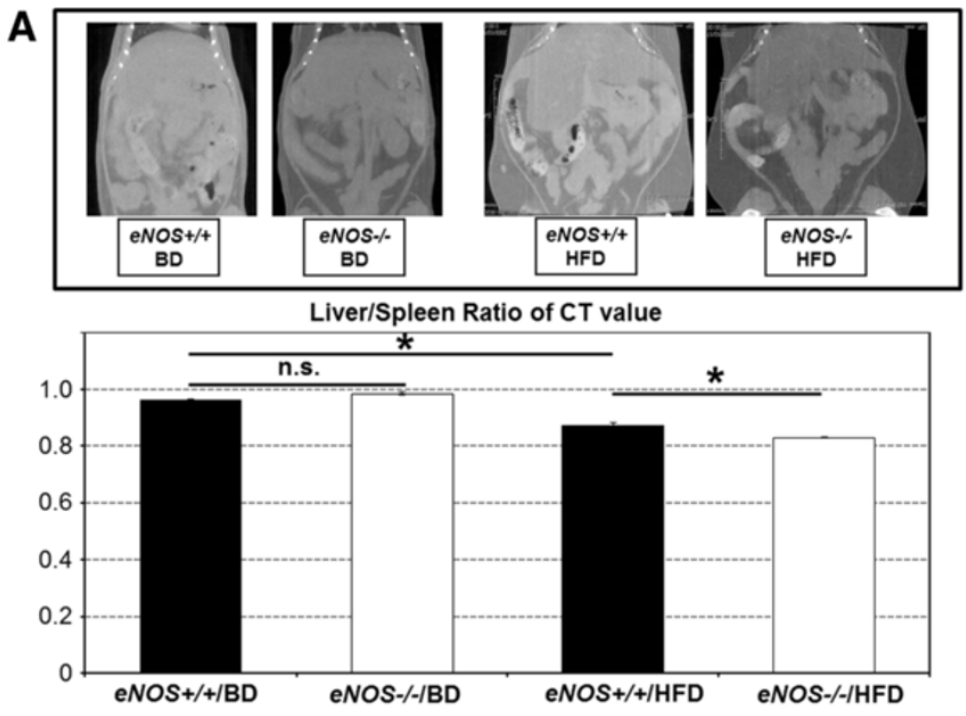

B
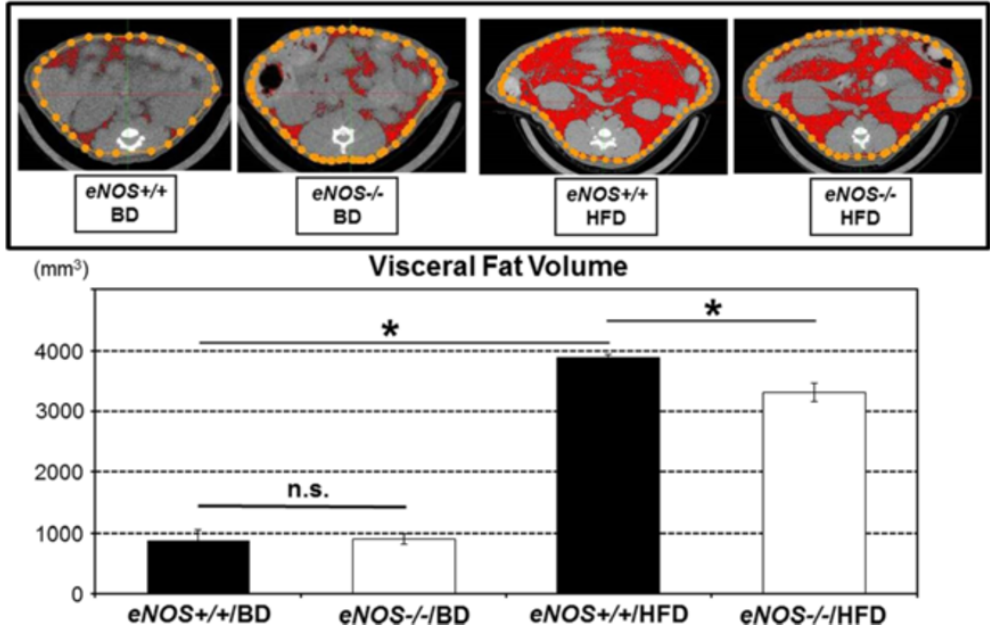

Fig. 1 Analysis of liver weight, lipid deposits in the liver, and visceral fat volume. a The liver-to-spleen attenuation ratio obtained using non-contrast CT images showed that hepatic steatosis was significantly severer in the eNOS-/-/HFD mice than in the eNOS+/+/HFD mice at 12 weeks. $\mathbf{b}$ The visceral fat volume quantified in the CT imaging of the eNOS-/-/HFD mice was significantly lower than that of the eNOS+/+/HFD mice at 12 weeks. (Data were expressed as the mean \pm SEM. ${ }^{*} P<0.05$, represents a significant difference; $n$. s. represents no significant difference.)

been described in several studies [25, 26]. Moreover, another study demonstrated that key signaling molecules mediating the metabolic actions of insulin were necessary for insulin to stimulate the production of NO and vasodilatation in human vascular endothelium [27]. Thus, we focused on the role of the eNOS gene in the pathogenesis of NAFLD/NASH; NO, produced by various isoforms of NOS, is an ubiquitous signaling molecule involved in the regulation of metabolic homeostasis, and eNOS, which the endothelium produces as a vasoactive substance, has been shown to serve important functions, including the regulation of regional blood flow, insulin resistance, and energy production $[6,28]$.
In the present study, we used an HFD-induced NAFLD/NASH mouse model with or without the eNOS gene. In our NAFLD/NASH model using HFD conditions, prominent hepatic steatosis and very mild liver inflammation were observed after 12 weeks, but typical fibrosis was not observed. In this "early-stage NAFLD/ NASH" model, eNOS-derived NO changed the fat distributions in the liver and viscera of the mice. eNOSknockout mice reportedly exhibit a clustering of symptoms belonging to the metabolic syndrome phenotype, such as body weight gain, hypertension, insulin resistance, and dyslipidemia [29]. These parameters of eNOS-knockout mice and wild-type mice reportedly differ according 


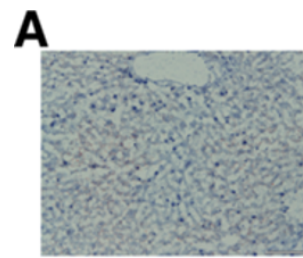

eNOS+/+/BD

B

C

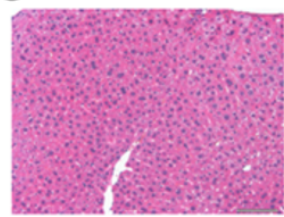

eNOS+/+/BD

D

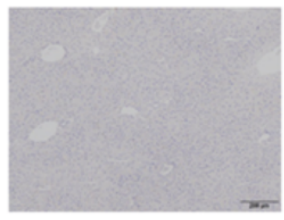

eNOS+/+/BD

E

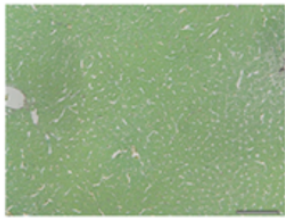

eNOS+/+/BD

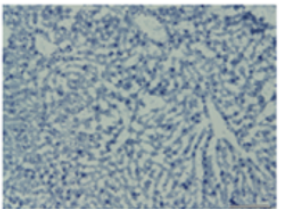

eNOS-/-/BD

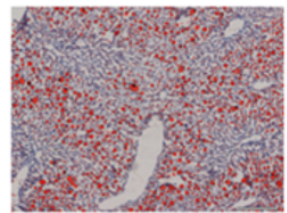

eNOS+/+/HFD
eNOS-/-/HFD

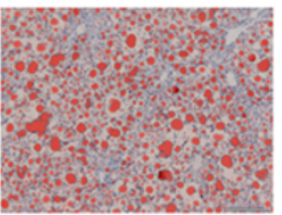

eNOS+/t eNOS-\%

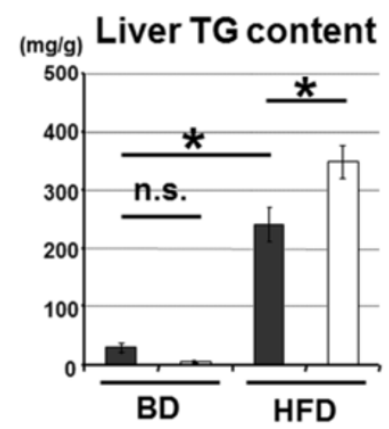

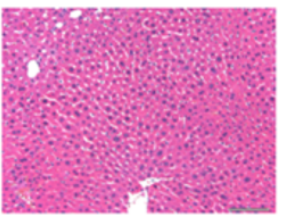

eNOS-/-/BD

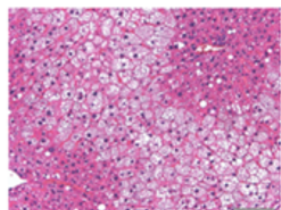

eNOS+/+/HFD

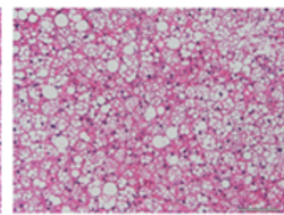

eNOS-/-/HFD

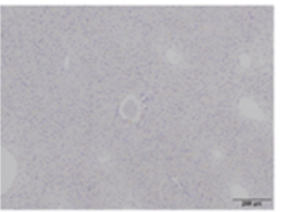

eNOS-/-/BD

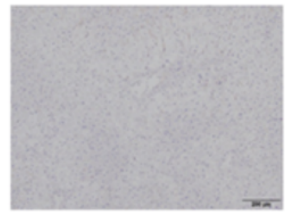

eNOS+/+/HFD

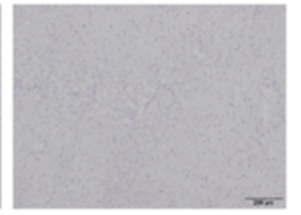

eNOS-//HFD

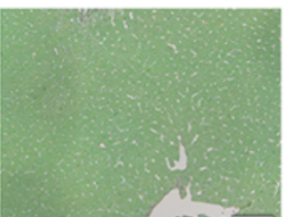

eNOS-/-/BD

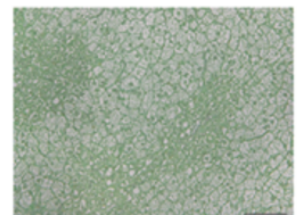

eNOS+/+/HFD

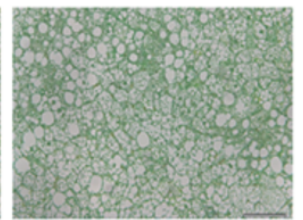

eNOS-/-/HFD

Fig. 2 Analysis of liver steatosis and inflammation. a Oil-red O staining (red color) shows lipid deposits in liver samples. At 12 weeks, macrovesicular steatosis was visible in the eNOS-///HFD mice and microvesicular steatosis was visible in the eNOS+/+/HFD mice. Scale bar, $200 \mu \mathrm{m}$. $\mathbf{b}$ The liver TG content was significantly higher in the eNOS-///HFD mice than in the eNOS+/+/HFD mice. The liver TG content was significantly higher in the eNOS +/+/HFD mice than in the eNOS+/+/BD mice, which were regarded as the control group. c Liver samples stained using H\&E. An evaluation of the number of inflammatory foci per field and the amount of ballooning revealed that the eNOS-/-/HFD mice exhibited severer liver inflammation than the eNOS+/+/HFD mice at 12 weeks. Scale bar, $200 \mu \mathrm{m}$. $\mathbf{d}$ and e Liver samples stained using myeloperoxidase (d) and Sirius red (e) showed no significant positive findings. (Data were expressed as the mean \pm SEM. ${ }^{*} P<0.05$, represents a significant difference; n.s. represents no significant difference.) 


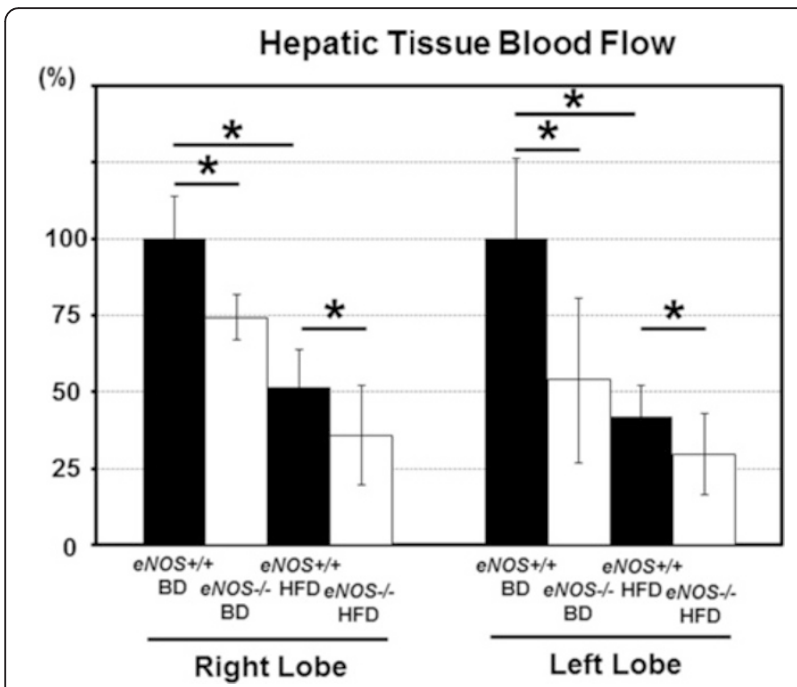

Fig. 3 Analysis of hepatic talysis of hepatic tissue blood flow. The hepatic tissue blood flow measured using a noncontact-type laser Doppler blood flow meter was significantly lower in the eNOS-/mice than in the eNOS+/+ mice under each feeding condition (BD or HFD) in both hepatic lobes at 12 weeks. The hepatic tissue blood flow of the eNOS+/+/HFD mice was significantly lower than that of the eNOS+/+/BD mice. (Data were expressed as the mean \pm SEM. ${ }^{*} P$ $<0.05$ represents a significant difference.)

to diet, sex, and the study period [28-34]. In both the eNOS-knockout mice and the wild-type mice fed an HFD during the 12 weeks of our study period, obesity, insulin resistance, dyslipidemia and high serum leptin levels were observed, although these markers were comparable between the eNOS-knockout and the wild-type mice fed an HFD, with the exception of the serum cholesterol level. Hepatic steatosis and the increases in the serum ALT levels were significantly severer in the eNOS-knockout mice fed an HFD than in the wild-type mice fed an HFD, while the visceral fat volume was significantly lower in the eNOS-knockout mice fed an HFD, compared with the wild-type mice fed an HFD in this study.

The mechanism responsible for the changes in fat distribution in the liver and viscera of eNOS-knockout mice fed an HFD may be associated with the hepatic blood flow. Actually, eNOS-knockout mice fed an HFD exhibited a significantly lower hepatic tissue blood flow, compared with wild-type mice fed an HFD, with no significant difference in systemic insulin clearance observed between the eNOS-knockout mice and the wild-type mice fed an HFD. These results suggested that eNOS may play an important role in the progression of "earlystage NAFLD/NASH" not through insulin resistance, but through direct hepatic vascular action under the HFD condition. An analysis of factors associated with lipid metabolism in the liver showed that the liver MTP activity level was significantly lower in the eNOS-knockout mice fed an HFD than in the wild-type mice fed an
HFD. MTP is a heterodimeric lipid transfer protein that is essential for very low density lipoprotein synthesis and transfer; a polymorphism of the MTP promoter reportedly leads to decreased MTP transcription and a greater intrahepatocellular triglyceride accumulation, thereby determining the susceptibility to NASH [35]. The downregulation of hepatic MTP activity could lead to a decreased visceral fat volume by decreasing the outflow of lipids from the liver. However, we could not determine the relation between eNOS and the liver MTP activity in our study model, and few studies have examined this point.

eNOS is known to serve important functions, including the regulation of vascular tone and regional blood flow [6]. On the other hand, hepatic tissue blood flow and hepatic microcirculation have been shown to be strongly associated with the progression of NAFLD/ NASH [7, 36-38]. In NASH liver, ballooning, which consists of fat-laden swollen hepatocytes, causes sinusoidal distortion, reducing the intrasinusoidal volume and microvascular blood flow [36-38]. In our eNOS-knockout mouse model, while wild-type mice fed an HFD had a significantly lower hepatic tissue blood flow than the wild-type mice fed a BD, the eNOS-knockout mice fed a $\mathrm{BD}$ had a significantly lower hepatic blood flow than the wild-type mice fed a BD. These results suggested that the decrease in hepatic blood flow in the eNOS-knockout mice fed an HFD was not only a secondary effect of lipid accumulation and compression of the sinusoid, but was also caused by the eNOS gene deficiency.

Sheldon et al. reported that chronic NOS inhibition via $\mathrm{N}^{\omega}$-nitro-L-arginine methyl ester in obese Otsuka LongEvans Tokushima Fatty rats reduced hepatic mitochondrial respiration, leading to increased hepatic triacylglycerol accumulation, and increased hepatic inflammation, although the specific mechanism remained unclear [8]. They did not examine blood flow in the hepatic tissue; however, the mechanism related to exacerbated earlystage NAFLD pathogenesis under the condition of eNOS deficiency might be associated with the function of hepatic mitochondrial respiration.

Regarding insulin resistance, no significant differences in systemic insulin resistance, such as the HOMA-IR or ITT results, were observed between the wild-type and eNOS-knockout mice under the HFD conditions in this model. However, direct hepatic insulin responsiveness was not assessed in this study.

Although the present study revealed a change in the fat distribution induced by both the eNOS gene and the HFD in mice in an "early-stage NAFLD/NASH" model, longer-term analysis may detect a different phenomenon in "advanced-stage NAFLD/NASH." A potential limitation of the current study includes the use of systemic eNOS-knockout mice. In particular, 

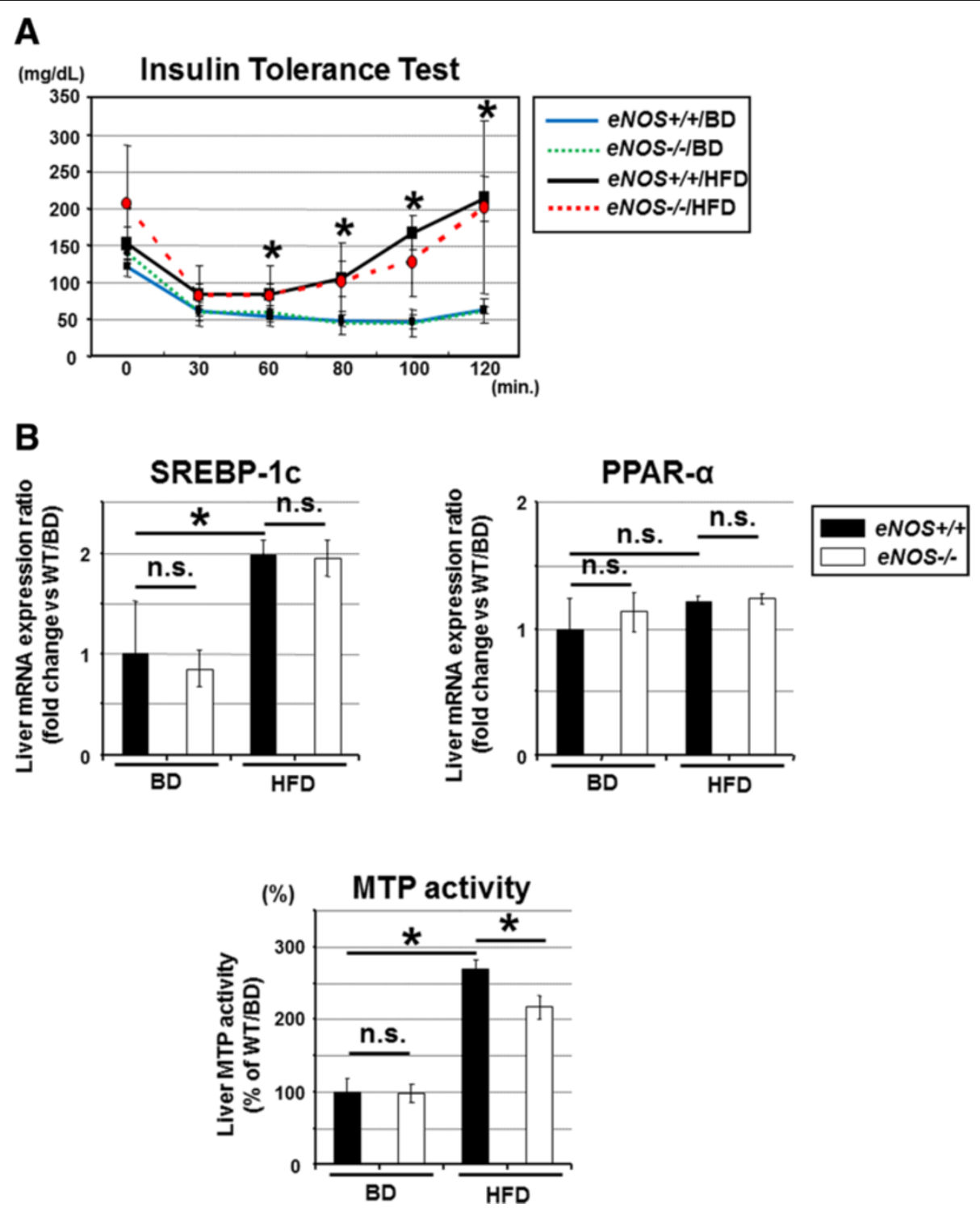

Fig. 4 Analysis of the mechanism associated with the pathogenesis of NAFLD. a ITT results at 12 weeks showed no significant difference in the systemic response to insulin injection between the eNOS+/+/HFD mice and the eNOS-/-/HFD mice, while it was significantly lower in the eNOS +/+/HFD mice than in the eNOS+/+/BD mice, which were regarded as the control group. $\mathbf{b}$ The liver SREBP-1c and PPAR-a1 mRNA expression levels were comparable between the eNOS-/-/HFD mice and the eNOS-/-/HFD mice. The liver MTP activity level was significantly lower in the eNOS-///HFD mice than in the eNOS+/+/HFD mice at 12 weeks. The liver SREBP-1c mRNA expression level and the liver MTP activity level were significantly higher in the $e N O S+/+/ H F D$ mice than in the eNOS+/+/BD mice, which were regarded as the control group. (Data were expressed as the mean \pm SEM. ${ }^{*} P<0.05$, represents a significant difference; $n . s$. represents no significant difference.)

studies using liver-specific eNOS-knockout mice fed an HFD or eNOS transgenic mice fed an HFD in a long-term HFD feeding analysis are needed. Further studies are needed to verify hepatic mitochondrial respiration markers and hepatic inflammation states, and to examine the relationship between the changes in hepatic blood flow and the degree of liver injury in order to determine the exact underlying mechanism.

\section{Conclusions}

In conclusion, a deficiency of eNOS-derived NO may change the fat distributions in the liver and viscera, thereby promoting the progression of disease in an HFD-induced, early-stage NASH mouse model by changing the hepatic tissue blood flow. This report is also the first study to examine the fat distribution and the pathogenesis of NAFLD/NASH using an imaging procedure 


\section{in an NAFLD/NASH mouse model with or without the eNOS gene.}

\begin{abstract}
Abbreviations
ALT: alanine aminotransferase; BD: basal diet; Chol: cholesterol; CT: computed tomography; eNOS: endothelial nitric oxide synthase; HFD: high-fat diet; HOMA-IR: homeostasis model assessment of IR; iNOS: inducible nitric oxide synthase; IR: insulin resistance; ITT: insulin tolerance test; MPO: myeloperoxidase; MTP: microsomal triglyceride transfer protein; NAFL: non-alcoholic fatty liver; NAFLD: non-alcoholic fatty liver disease; NAS: NAFLD activity score; NASH: non-alcoholic steatohepatitis: NEFA: nonesterified fatty acid; nNOS: neuronal nitric oxide synthase; NO: nitric oxide; NOS: nitric oxide synthase; PPAR-a1: peroxisome proliferators-activated receptor alpha1; SREBP-1c: sterol regulatory element binding protein-1c; TG: triglyceride.
\end{abstract}

\section{Competing interests}

None of the co-authors has any conflict of interest to declare concerning the material presented in this manuscript.

\section{Authors' contributions}

AN, YN, KF, and MY conceived of the study, and participated in its design and coordination. $\mathrm{KI}$ and $\mathrm{YO}$ participated in the design of the study and performed the statistical analysis. YN, KF, TK, YS, and YN performed the experiments and analyzed the data. KW, MN, SS, NM, and YT were involved in drafting the manuscript. AN and $\mathrm{YN}$ wrote the paper. All the authors have read and approved the final manuscript.

\section{Acknowledgments}

This study was supported in part by a grant from the National Institute of Biomedical Innovation and a grant program entitled "Collaborative Development of Innovative Seeds" from the Japan Science and Technology Agency to A.N., and a grant for National Center for Global Health and Medicine (26A-107) to Y.N

The skillful technical assistance of Machiko Hiraga, Tamiyo Taniguchi, and Shizuko Kobayashi is gratefully acknowledged.

\section{Author details}

'Department of Gastroenterology, Yokohama City University Graduate School of Medicine, 3-9 Fuku-ura, Kanazawa-ku, 236-0004 Yokohama, Kanagawa, Japan. ${ }^{2}$ Department of Gastroenterology, National Center for Global Health and Medicine, 1-21-1, Toyama, Shinjuku-ku, 162-8655 Tokyo, Japan. ${ }^{3}$ Department of Pharmacology, Graduate School of Dentistry, Osaka University, 1-8 Yamadaoka, 565-0871 Suita, Osaka, Japan. ${ }^{4}$ Department of Gastroenterology, Kyushu Medical Center, National Hospital Organization, 1-8-1, Jigyohama, Chuo-ku, 810-8563 Fukuoka, Japan. ${ }^{5}$ The Research Center for Hepatitis and Immunology, National Center for Global Health and Medicine, 1-7-1, Konodai, 272-8516 Ichikawa, China, Japan. 'Department of Molecular Pathology, Yokohama City University Graduate School of Medicine, 3-9 Fuku-ura, Kanazawa-ku, 236-0004 Yokohama, Kanagawa, Japan. ${ }^{7}$ Department of Surgical Pathology, Tokyo Women's Medical University, 8-1, Kawada-cho, Shinjuku-ku, Tokyo 162-8666, Japan. ${ }^{8}$ Department of Endocrinology and Metabolism, Yokohama City University Graduate School of Medicine, 3-9 Fuku-ura, Kanazawa-ku, 236-0004 Yokohama, Kanagawa, Japan.

Received: 20 June 2015 Accepted: 7 December 2015 Published online: 17 December 2015

\section{References}

1. Day CP, James OF. Steatohepatitis: a tale of two "hits"? Gastroenterology. 1998;114:842-5

2. Fujita K, Nozaki Y, Wada K, Yoneda M, Fujimoto Y, Fujitake M, et al. Dysfunctional very-low-density lipoprotein synthesis and release is a key factor in nonalcoholic steatohepatitis pathogenesis. Hepatology. 2009;50: $772-80$

3. Imajo K, Fujita K, Yoneda M, Nozaki Y, Ogawa Y, Shinohara Y, et al. Hyperresponsivity to Low-Dose Endotoxin during Progression to Nonalcoholic Steatohepatitis Is Regulated by Leptin-Mediated Signaling. Cell Metab. 2012;3(16):44-54.
4. Nozaki Y, Fujita K, Yoneda M, Wada K, Shinohara Y, Takahashi H, et al. Long-term combination therapy of ezetimibe and acarbose for non-alcoholic fatty liver disease. J Hepatol. 2009;51:548-56.

5. Alderton WK, Cooper CE, Knowles RG. Nitric oxide synthase: structure, function and inhibition. Biochem J. 2001;357:593-615.

6. Huang PL. eNOS, metabolic syndrome and cardiovascular disease. Trends Endocrinol Metab. 2009;20:295-302.

7. McCuskey RS, Ito Y, Robertson GR, McCuskey MK, Perry M, Farrell GC. Hepatic microvascular dysfunction during evolution of dietary steatohepatitis in mice. Hepatology. 2004;40:386-93.

8. Sheldon RD, Padilla J, Jenkins NT, Laughlin MH, Rector RS. Chronic NOS inhibition accelerates NAFLD progression in an obese rat model. Am J Physiol Gastrointest Liver Physiol. 2015;15(308):G540-9.

9. Toshima G, Iwama Y, Kimura F, Matsumoto Y, Miura M, Takahashi J, et al. LipoSERCH ${ }^{\oplus}$; Analytical GP-HPLC method for lipoprotein profiling and its applications. J Biol Macromol. 2013;13:21-32.

10. Usui S, Hara Y, Hosaki S, Okazaki M. A new on-line dual enzymatic method for simultaneous quantification of cholesterol and triglycerides in lipoproteins by HPLC. J Lipid Res. 2002;43:805-14.

11. Terauchi Y, Takamoto I, Kubota N, Matsui J, Suzuki R, Komeda K, et al. Glucokinase and IRS-2 are required for compensatory beta cell hyperplasia in response to high-fat diet-induced insulin resistance. J Clin Invest. 2007; 117:246-57.

12. Lettéron $\mathrm{P}$, Sutton $\mathrm{A}$, Mansouri A, Fromenty B, Pessayre D. Inhibition of microsomal triglyceride transfer protein: another mechanism for drug-induced steatosis in mice. Hepatology. 2003;38:133-40.

13. Perlemuter G, Sabile A, Letteron P, Vona G, Topilco A, Chrétien Y, et al. Hepatitis $C$ virus core protein inhibits microsomal triglyceride transfer protein activity and very low density lipoprotein secretion: a model of viral-related steatosis. FASEB J. 2002;16:185-94.

14. Theeraladanon C, Takahashi N, Shiina M, Hamada K, Takada Y, Endo H, et al. Rational approach to the synthesis, evaluation, and (68)ga labeling of a novel 4-anilinoquinoline epidermal growth factor receptor inhibitor as a new imaging agent that selectively targets the epidermal growth factor receptor tyrosine kinase. Cancer Biother Radiopharm. 2010;25:479-85.

15. Park SH, Kim PN, Kim KW, Lee SW, Yoon SE, Park SW, et al. Macrovesicular hepatic steatosis in living liver donors: use of $\mathrm{CT}$ for quantitative and qualitative assessment. Radiology. 2006;239:105-12.

16. Judex S, Luu YK, Ozcivici E, Adler B, Lublinsky S, Rubin CT. Quantification of adiposity in small rodents using micro-CT. Methods. 2010:50:14-9.

17. Eguchi Y, Mizuta T, Sumida Y, Ishibashi E, Kitajima Y, Isoda H, et al. The pathological role of visceral fat accumulation in steatosis, inflammation, and progression of nonalcoholic fatty liver disease. J Gastroenterol. 2011;46 Suppl 1:70-8.

18. Luu YK, Lublinsky S, Ozcivici E, Capilla E, Pessin JE, Rubin CT, et al. In vivo quantification of subcutaneous and visceral adiposity by micro-computed tomography in a small animal model. Med Eng Phys. 2009:31:34-41.

19. Ishikawa H, Jin MB, Ogata T, Taniguchi M, Suzuki T, Shimamura T, et al. Role of cyclic nucleotides in ischemia and reperfusion injury of canine livers. Transplantation. 2002;73:1041-8.

20. Masuko H, Jin MB, Horiuchi H, Suzuki T, Taniguchi M, Shimamura T, et al. Protective effect of agiotensin || type I receptor antagonist, CV-11974, on ischemia and reperfusion injury of the liver. Transplantation. 2001;71:1034-9.

21. Khandoga A, Huettinger S, Khandoga AG, Li H, Butz S, Jauch KW, et al. Leukocyte transmigration in inflamed liver: A role for endothelial cellselective adhesion molecule. J Hepatol. 2009;50:755-65.

22. Kleiner DE, Brunt EM, Van Natta M, Behling C, Contos MJ, Cummings OW, et al. Design and validation of a histological scoring system for nonalcoholic fatty liver disease. Hepatology. 2005;41:1313-21.

23. Fujita K, Nozaki Y, Wada K, Yoneda M, Endo H, Takahashi H, et al. Effectiveness of antiplatelet drugs against experimental non-alcoholic fatty liver disease. Gut. 2008:57:1583-91.

24. Tilg $\mathrm{H}$, Moschen AR. Evolution of inflammation in nonalcoholic fatty liver disease: the multiple parallel hits hypothesis. Hepatology. 2010;52:1836-46.

25. Seifalian AM, El-Desoky A, Davidson BR. Hepatic indocyanine green uptake and excretion in a rabbit model of steatosis. Eur Surg Res. 2001;33:193-201.

26. Shigefuku R, Takahashi H, Kobayashi M, Ikeda H, Matsunaga K, Okuse C, et al. Pathophysiological analysis of nonalcoholic fatty liver disease by evaluation of fatty liver changes and blood flow using xenon computed tomography: can early-stage nonalcoholic steatohepatitis be distinguished from simple steatosis? J Gastroenterol. 2012;47:1238-47. 
27. Zeng G, Quon MJ. Insulin-stimulated production of nitric oxide is inhibited by wortmannin. Direct measurement in vascular endothelial cells. J Clin Invest. 1996;98:894-8.

28. Le Gouill E, Jimenez M, Binnert C, Jayet PY, Thalmann S, Nicod P, et al. Endothelial nitric oxide synthase (eNOS) knockout mice have defective mitochondrial beta-oxidation. Diabetes. 2007;56:2690-6.

29. Nisoli E, Clementi E, Carruba MO, Moncada S. Defective mitochondrial biogenesis: a hallmark of the high cardiovascular risk in the metabolic syndrome? Circ Res. 2007;100:795-806.

30. Cook S, Hugli O, Egli M, Vollenweider P, Burcelin R, Nicod P, et al. Clustering of cardiovascular risk factors mimicking the human metabolic syndrome $X$ in eNOS null mice. Swiss Med Wkly. 2003;133:360-3.

31. Duplain H, Burcelin R, Sartori C, Cook S, Egli M, Lepori M, et al. Insulin resistance, hyperlipidemia, and hypertension in mice lacking endothelial nitric oxide synthase. Circulation. 2001:104:342-5.

32. Durand JL, Nawrocki AR, Scherer PE, Jelicks LA. Gender differences in adiponectin modulation of cardiac remodeling in mice deficient in endothelial nitric oxide synthase. J Cell Biochem. 2012;113:3276-87.

33. Mohan S, Reddick RL, Musi N, Horn DA, Yan B, Prihoda TJ, et al. Diabetic eNOS knockout mice develop distinct macro- and microvascular complications. Lab Invest. 2008;88:515-28.

34. Nisoli E, Clementi E, Paolucci C, Cozzi V, Tonello C, Sciorati C, et al. Mitochondrial biogenesis in mammals: the role of endogenous nitric oxide. Science. 2003;299:896-9.

35. Namikawa C, Shu-Ping Z, Vyselaar JR, Nozaki Y, Nemoto Y, Ono M, et al. Polymorphisms of microsomal triglyceride transfer protein gene and manganese superoxide dismutase gene in non-alcoholic steatohepatitis. J Hepatol. 2004;40:781-6.

36. Farrell GC, Teoh NC, McCuskey RS. Hepatic microcirculation in fatty liver disease. Anat Rec (Hoboken). 2008;291:684-92.

37. Oda M, Yokomori H, Han JY. Regulatory mechanisms of hepatic microcirculation. Clin Hemorheol Microcirc. 2003;29:167-82. Review.

38. Rosenstengel S, Stoeppeler S, Bahde R, Spiegel HU, Palmes D. Type of steatosis influences microcirculation and fibrogenesis in different rat strains. J Invest Surg. 2011;24:273-82.

\section{Submit your next manuscript to BioMed Central and we will help you at every step:}

- We accept pre-submission inquiries

- Our selector tool helps you to find the most relevant journal

- We provide round the clock customer support

- Convenient online submission

- Thorough peer review

- Inclusion in PubMed and all major indexing services

- Maximum visibility for your research

Submit your manuscript at www.biomedcentral.com/submit 